\title{
Research on the High Performance System Development and Cloud Platform Building Mode under Parallel Framework
}

\author{
Xinxin Xie ${ }^{1, a}$ and Wenzhun Huang ${ }^{1, b^{*}}$ \\ ${ }^{1}$ School of Information Engineering, Xijing University, Xi'an 710123, China \\ a346148500@qq.com, bhuangwenzhun@xijing.edu.cn \\ ${ }^{*}$ The corresponding author
}

\begin{abstract}
Keywords: High performance; Cloud platform; Parallel framework; Development platform
\end{abstract}
\begin{abstract}
In this paper, we conduct research on the high performance system development and basic cloud platform building model under parallel framework. Cloud computing is a kind of the public service, its security problem is complex and requires skill, standard, regulation, law and so on various aspects, multi-pronged jointly solve security problems. From a technical level, to improve the security of cloud computing technology research and development is the key point to solve the problem of cloud computing security, protection of identity protection, infrastructure and information data from three aspects, such as protection of the cloud computing security technology research will effectively promote cloud computing security problem. Under this historical background, we propose the novel parallel framework for cloud platform that is innovative.
\end{abstract}

\section{Introduction}

Development of high performance computing also has two ways, one way is based on the existing semiconductor integrated circuit technology, the microprocessor technology, through the ability to improve the core parallel processing implementation and another way is to break through the silicon semiconductor devices, the development of new technology, traditional, including superconducting computing, quantum computing, biology, computing and optical computing, etc.

Use parallel computing to solve the problem of basic general process for: for a given application problems, first of all, computational scientists to apply this into a numerical or numerical computing problems; Then computer scientists design parallel algorithm for this problem, and through some kind of parallel programming language to achieve it; The experts in the field of application in some specific parallel computer to run on application software to solve this problem. The development of parallel computing and computer science in many fields, in addition to the law of the development of the discipline itself that has also been a great influence in the industry, recently, with the continuous development of hardware technology and new applications, parallel computing also had some new development, such as multi-core architecture cloud computing, personal high performance computer.

According to the literature survey, the developmental trend of the issues could be summarized as the follows. (1) Data-centric cloud computing. It is a kind of new method of the computing resource sharing, to huge system together is to provide a variety of computing services. Many factors driving the demand for this kind of environment, including the general development of the Internet and the development of mature, mobile devices, the popularity of search engines, social networks and mobile business, etc. (2) To popularize and apply for the purpose of personal high performance computer. Technology appears with the mainstream, and the parallel computing architecture, parallel algorithm, parallel programming, and the application of parallel studies have produced important influence, has brought new challenges. (3) Mainstream for general multi-core architecture. Technology is in same processor integrating two or more complete calculation kernel, each computing kernel in essence is relatively simple microprocessor. Multiple computing kernels can be executed in parallel instruction, so as to realize a chip inside the thread level parallelism, and more tasks can be performed in a specific time implementation tasks in parallel, and thus improve the computing power [1-3]. 
The next generation of intelligent network with flexible network architecture and open ability, support for multiple network, multi-protocol access, realize business and the separation agreement, achieve business open, network integration. Its evolution analysis from the following several aspects: evolution of a business function evolution, protocol, network architecture and platform. Based on the cloud platform of next generation of intelligent network based on the platform of SNE, construction of ability of reusable business component warehouse, to integrate the existing business into a unified independent delivery business, and support the expansion of the domain specific solutions.

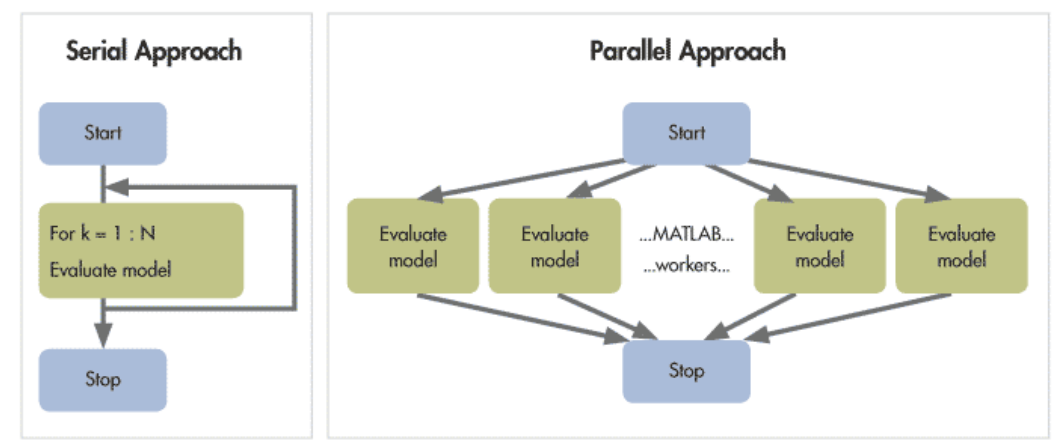

Figure 1. The Parallel Computing Framework and Primary Architecture

In this article, we conduct research on the high performance system development and basic cloud platform building model under parallel framework. Cloud computing is a hot spot in the research of the information explosion era, whether it is a traditional enterprise, or Internet companies pay more and more attention to the various related data analysis and use of, how to handle huge amounts of data has become one of the Internet enterprise's core competitiveness, the open source implementation of cloud computing and its high fault tolerance, cross-platform, and many other advantages, to quickly get the favor of many enterprises. In the later sections, we will discuss the issues in detail.

\section{The Proposed Methodology}

The General Features of the Cloud Computing. Cloud computing is a way of sharing the IT infrastructure that can connect pool huge system providing IT services, make the operation of the enterprise data center is more similar to the Internet, through the way of safety and extensible like the virtual resources, computing resources can be accessed and Shared. Using virtualization technology existing server can be more granular computing and core storage capacity of cutting to improve the efficiency of the use of hardware resources. On the other hand also for large scale cloud computing integration provides a common interface standard.

Cloud computing make originally IT industry hardware and software products into the web-based services, created a new market space. Focus on cloud computing is growing at home and abroad, the global cloud computing is also in the rapidly expanding market scale, cloud computing services since produced that already have many users, accordingly, the challenges arise as the follows.

- Cloud platform ability is at risk of abuse. Once the one or some nodes in the cloud is hacked, a hacker could continue to attack by these nodes and controls the cloud computing platform, the cloud computing platform is ultimately controlled by organized by super computing power to implement network disruption and attacks.

- Cloud computing security solutions provide new development ideas. Cloud computing is not only increases the safety hidden trouble, in fact the cloud itself also offers a new way for the security policy [4].

- Cloud computing data security hidden danger. Domestic data center operations or core basic services such as web hosting, that room rental is given priority to, the safety of the user data protection and privacy protection is still unable to meet the future demand for the development of cloud computing. 
- Cloud computing is different from traditional IT business, and traditional data center has vastly different. The use of virtualization technology, make as many as thousands of different regions in the server together build up a data interaction platform for cloud computing service.

Linux load balancing cluster building successful, using test found that heart rate and failover. When the server node failure when it can be removed from the cluster; Later, when the fault is ruled out automatically to rejoin the cluster nodes in the pool in the process of this change is a dynamic adaptive load balancing strategy. The basic principle is that by making the calculation of distribution on a large number of the distributed computers, rather than the local computer or remote server, the operation of the enterprise data center will be more similar to the Internet. This enables the enterprise to the resources to switch to the need of application, access to the computer and storage system based on the requirements [5].

The Parallel Computing Framework. Accompanied with the general process of digitization, data processing, storage, and transmission has been rapid development. The demand of the high bandwidth short interconnection has become the bottleneck of system development. Affected by factors such as loss and crosstalk, based on copper wire electrical interconnection transmission distance under the circumstance of high bandwidth has been curbed, the cost also rose and too much cable also increases the complexity of the system and weight of the wiring. Compared with the electrical interconnection, optical interconnection based on multimode fiber has high bandwidth, low loss, no crosstalk and matching, and the problem of electromagnetic compatibility and widely used in cabinets, framework between the high-speed interconnection between the room and board.

In order to accelerate convergence, he technical means such as multiple grid method is used to improve computational efficiency as a whole. Automatic dividing and computing grid is to achieve most direct and most effective coarse-grained parallel task allocation strategy, can reduce redundant computation, correctly mapped to the parallel processing system, ensure the load balancing, to ensure the scalability of the primary algorithm paradigm.

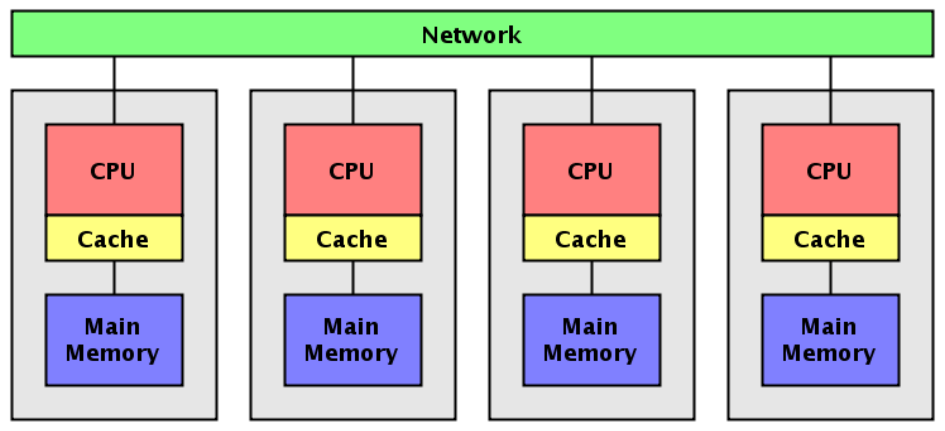

Figure 2. The Parallel Computing Framework

Parallel computing, as a kind of to a computational task allocation to multiple processors and the calculation method of running at the same time, can largely to speed up the process of the rough set attribute reduction, the classification of the most important attribute selection process as follows.

$$
\gamma(A, D)=\left[\sum_{j=1}^{|U|}\left(V_{i=1}^{P} R\left(d_{i x}\right)\right)\right] /[U]
$$

Parallel optical interconnection through multiple root parallel light transmission as can be realized under the rate of high bit rate relatively long distance transmission, which is why the one cause of the parallel optical interconnection. Therefore, the revised standard could be summarized as follows.

$$
R_{j k}=\exp \left(-\frac{\left\|x_{j}-x_{k}\right\|^{2}}{2 \sigma}\right)
$$

Load balancing cluster the overall load of system can be reasonably allocated to each node, make each node will not collapse because overworked and the overall system load capacity, load demand is greater than the system as a whole can increase system node, expand smoothly system load capacity. 
Supercomputing cluster is through connects multiple machines at the same time dealing with complex computational problems of computer. For solving the problem will be divided into several parts, each part by an independent processor for processing. Inspired by parallel computing ideas, first calculates the process of attribute importance distribution operations on multiple processors at the same time, put all the calculation results and the basic combination of selected from one of the most important attribute, it largely reduce the time consumption of the proposed algorithm [6].

The High Performance Computing. High performance computing is a novel branch of computer science, research and development related software in general parallel algorithm, committed to the development of high performance computer. With the rapid development of information society, the high performance computing has become after the pure science and experimental science the third pillar of scientific research. Network will be the most important trend to HPC as the grid has become a high performance computing a new research hotspot. Grid will be distributed in the computer, data, storage devices, users and software organization into a logical whole, industries can run on the basis of their respective application based primary grid.

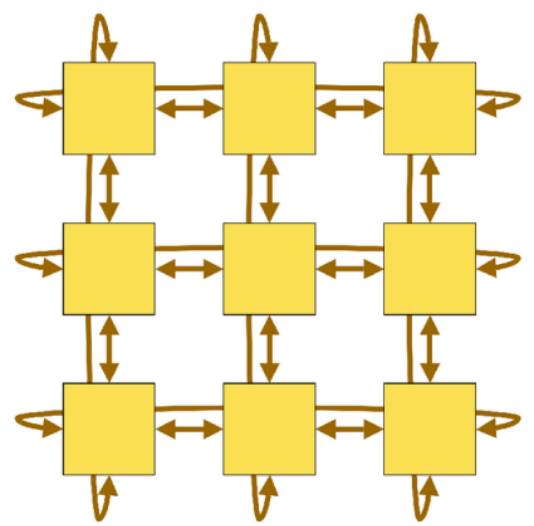

Figure 3. The High Performance Conputing System Chip Architecture and Pattern

To improve computing performance can be viewed from individual different directions, namely bottom-up or the top-down. The former is committed to developing high performance devices or the components, such as the quantum devices, superconducting chip, optical interconnection and optical storage components, biological molecular components, mainly in hardware aspects and the latter is dedicated to research in computational model and algorithm design. Custom computing system also needs a complete software environment, the application of computing research custom software, interface protocols and made up of their operating environment, special programming language that should be separated into the following procedures.

- In line with the now popular general characteristics in a high-level language. It has a good ability of compiler optimization, has designed a large number of successful application in the actual application, so the custom calculations of the user interface will be as far as possible the adoption of serial program.

- Communication between support efficient parallel processing computers. Can the description of the algorithm is effective to the problems of the supercomputing, find out that part of the algorithm that can be executed in parallel, the communication with parallel programming language statements to organize communication of multiple processes and able to complete synchronization control [7].

- A parallel task of control statements, able to handle data in parallel at the same time. On customization of the high-performance computer hardware platform, supported languages are supposed to be super scientific computing applications have extremely high efficiency that can reflect the programming language being used in parallel to express on a super computing problem solving.

The Cloud Platform Building Mode. Hadoop task scheduling and resource management issues have been one of research hotspots in the field of Hadoop cloud computing framework. As the duty and 
ability of each node in the basic Hadoop demand difference, how for task assignment is the most appropriate resources and resource management optimization that has been the focus of the research object in this field. As mobile core network gradually to the next generation of IMS core network, the evolvement of the target architecture of mobile intelligent network will also with the development of the evolution of core network architecture and evolution, intelligent network platform based on IMS as IMS application server, the session initiation protocol based on TCP/IP host. Considering the existing mobile core network and IMS network will co-exist for a long time during a certain period, the next generation of intelligent network architecture must support both existing CAP in a mobile network, and support the SIP of IMS network, a fusion of the intelligent business platform. This is conducive to the smooth evolution of network, at the same time can protect existing investments to the greatest extent and maximize investment returns.

When the actual deployment, can according to the configuration of the machine to decide. With better performance for single machine configuration, can put all the components are deployed on the same machine. If single low performance configuration by cluster architecture, these components can be deployed separately to different machines, in order to achieve the optimal overall performance. In the figure 4, we illustrate the sample code for the proposed cloud platform building mode.

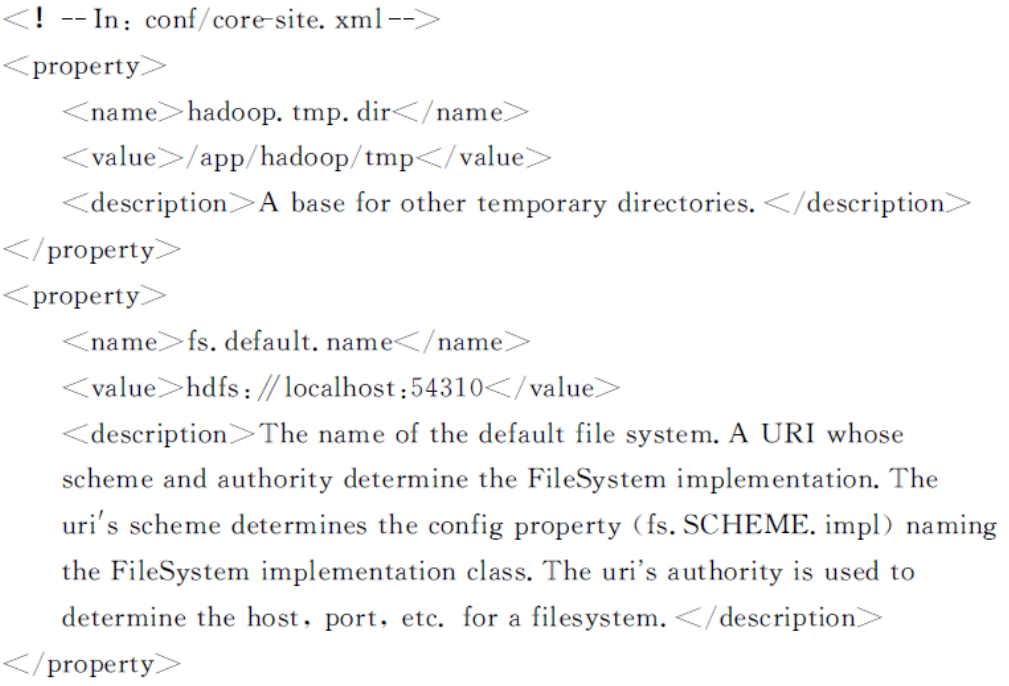

Figure 4. The Sample Code for the Cloud Platform Building Mode

\section{Summary}

In this paper, we conduct research on the high performance system development and cloud platform building model under parallel framework. In order to improve the tolerance of the high performance cluster computing system, checkpoints set become a widely used basic means. At present, a lot of checkpoints set up the coordination type set protocol, the protocol in the case of cluster scale expansion, caused huge system time synchronization overhead, and the implementation of blocking the normal calculation. Aiming at this problem, relate a coordinated checkpoint set protocol is used to eliminate the synchronous operation, using message log records to ensure system state consistency, and using the background thread implementation methods for the transparency setting. Based on the parallel framework, we optimize the traditional cloud platform with the high performance system that will be efficient. In the future, more related research will be finalized.

\section{References}

[1] Thorvaldsdóttir, Helga, James T. Robinson, and Jill P. Mesirov. Integrative Genomics Viewer (IGV): high-performance genomics data visualization and exploration. Briefings in bioinformatics 14.2 (2013): 178-192. 
[2] Kehoe, Rebecca R., and Patrick M. Wright. The impact of high-performance human resource practices on employees' attitudes and behaviors. Journal of Management 39.2 (2013): 366-391.

[3] Jeon, Nam Joong, et al. Compositional engineering of perovskite materials for high-performance solar cells. Nature 517.7535 (2015): 476-480.

[4] Qie, Long, et al. Synthesis of functionalized 3D hierarchical porous carbon for high-performance super capacitors. Energy \& Environmental Science 6.8 (2013): 2497-2504.

[5] Yang, Woon Seok, et al. High-performance photovoltaic perovskite layers fabricated through intermolecular exchange. Science 348.6240 (2015): 1234-1237.

[6] Aryee, Samuel, et al. Impact of high-performance work systems on individual-and branch-level performance: test of a multilevel model of intermediate linkages. Journal of Applied Psychology 97.2 (2012): 287.

[7] Lei, Ting, et al. A BDOPV - Based Donor - Acceptor Polymer for High - Performance n - Type and Oxygen - Doped Am bipolar Field - Effect Transistors. Advanced Materials 25.45 (2013): 6589-6593. 